\title{
A hybridization of SKH and RKFCM clustering optimization algorithm for efficient moving object exploration
}

\section{T. Mahalingam ${ }^{1}$}

Received: 1 June 2019 /Revised: 6 November 2020 / Accepted: 9 December 2020/ Published online: 17 February 2021

(C) The Author(s) 2021

\begin{abstract}
Object detection is a really crucial application of image processing. It is of essential value for object active surveillance and various other applications. Hence, the object detection has been extensively investigated. Refined Kernel fuzzy c-means system still carries a couple of downsides, for instance, to reduce the convergence level, obtaining stuck in the regional area minima and problem to initiation level of sensitiveness. To conquer the over problems, the following suggested strategy for stud krill herd Clustering Optimization Algorithm. This paper stands for an optimizing approach to global optimizing utilizing a unique variation of $\mathrm{KH}$ (Krill Herd). This approach is termed as Stud Krill Herd (SKH).The stud krill herd Clustering Optimization procedure utilizes to find the optimal centroid. At first, the background and foreground area partition is finished by hybridization of refined kernel fuzzy c means algorithm (RKFCM) with stud krill herd Clustering Optimization procedure. The suggested new strategy is scholarly and furthermore dynamic clustering system for dividing the moving object. This research study performs recommended a reliable object detection making use of hybridization of stud krill herd Clustering optimizing and RKFCM. Moving object tracing is done via the blob detection which happens under the tracing phase. The assessment phase has characteristic abstraction and categorization. High and appearance-based and quality based attributes are mined from fine-tuned frames whichever attended to classification. Considering that categorization we are developing usage of J48 (C4.5) i.e., decision tree based classification. The effectiveness of the advised method is analyzed through prior approaches $\mathrm{k}-\mathrm{NN}$ and MLP in regard to accuracy, f-measure, ROC and recall.
\end{abstract}

Keywords Refined kernel fuzzy c means $(\mathrm{RKFCM}) \cdot$ Krill herd · Stud krill herd · Decision tree · Genetic algorithm

T. Mahalingam

lingamrajthen@gmail.com

1 Sathyabama University, Tamil Nadu, India 


\section{Introduction}

Object tracing has become a strenuous research study subject because of enhanced need of smart security systems. Not just for surveillance, object discovery and also tracing are extensively utilized in activity categorization, attitude perception, multitude movement evaluation, communication between human-computer etc. Substantial progression is attained in object tracing throughout the here and now years and numerous standard procedures have been developed on object tracing $[4,5]$, attitude perception [31]. Still it remains to be a challenging problem as a consequence of the intricacies in a video series alike noise, unforeseeable movement of objects, fractional or complete occluding, lighting variant and backdrop variant. In which a video is caught by a movement video camera, both backdrop and forefront functions transform their location in every frameworks. Hence, segregation of backdrop and forefront comes to be extremely made complex task as foreground extrication utilizing conventional backdrop reduction or frame distinction does not use for such videos. Much, certain tasks have been stated to detect trace object/s efficiently in moving backdrop. A lot of the procedures essence forefront by calculating global movement and recompensing it by refining attribute factors [7, 27, 34, 42, 50].

Clustering is a procedure for identifying objects or patterns as if elements of the very same group are a lot more comparable to each other than elements coming from various groups. Several clustering approaches are utilized, these types of hard clustering strategy along with the fuzzy clustering strategy; all are which has its very specific unique features. The traditional hard clustering approach limits every factor of the information organized single cluster. Consequently, with this strategy the segment outcomes are frequently extremely crisp, i.e., all pixel of the image comes from precisely simply one class. In lots of genuine circumstances, for images, problems such as minimal spatial results, inadequate disparity, overlaying intensities, noise and intensity in uniformities variant make this hard (crisp) separation a complicated task.

The standard FCM procedure functions well on many clamorous images and have some severe constraint not integrate any type of details concerning spatial context, that create it to be sensible noise and imaging artefacts. Latterly, some strategies were suggested for raising the effectiveness of FCM to noise by precisely moderating the objective functionality. Li et al. [38] included this regularizing term right into the adaptive FCM (AFCM) procedure [61] to get over the noise level of sensitivity of AFCM procedure. The previous approaches are declared to be a lot more durable to noise, they reveal substantial computing intricacy. To make up for this downside of FCM, the noticeable method is to slick the image prior to segregation. The traditional smoothing filters can effect in drop of vital image information, specifically image borders or edges. The alternate method is not available to carefully regulate the compromise in among the slicking and clustering.

The traditional FCM procedure is noise delicate as a result of not considering the spatial info in the image. Here the author provides fuzzy c-means cluster separation process based upon customized member (MFCMp, q) that integrates spatial info right into the member feature for clustering. The spatial feature is the weighted aggregation of the member feature in the community of every pixel present. The fast MFCMp, q procedure (FMFCMp, q) which accelerates the convergent of MFCMp, q procedure is accomplished when the MFCMp, $\mathrm{q}$ procedure is activated by the rapid fuzzy c-means procedure through analytical histogram [37].

Lots of thresholding based separation methods have actually been stated in the research completed the previous couple of years. During that study M. Sezgin et al. [47] discovered this 
global histogram strategies are being typically utilized to figure out the limit in multilevel thresholding. This technique is affected a major disadvantage of rapid development in time intricacy and for this reason cannot be virtually encompassed multilevel thresholding complication. Lots of techniques possess been revealed in the research to enhance the effectiveness and decrease the time intricacy of multilevel thresholding approaches. Recursive algorithm Yin et al. [60] lower the extensive refinement time to figure out the maximum limit values with the aid of an appearance table. Whenever the variety of limit enhances the approach will once again be affected extensive computing time song et al. [51]. Within that to get rid of the aforementioned disputes, bio-inspired processing standards have been made use of for multilevel thresholding. Evolutional procedures alike Genetic procedures Lai et al. [35], ant nest optimisation Ye et al. [59], honey bee optimisation Horng et al. [26], particle swam optimisation (PSO) Gao et al. [19] and bacterial foraging procedure (BF) Sathya et al. [46] are efficiently utilized in the domain of image separation based upon multilevel. Amongst such procedures, GA, PSO and BF are the utmost popular. Genetic procedures are motivated through the evolutional concepts of usual choice that discovers the optimum limits efficiently. Current investigates Fogel [15] revealed genetic procedures have a propensity to assemble in the direction of regional optima instead than the global maximum value of the dispute that effects misstated of objects in separated image. Regarding the other hand, PSO revealed a couple of unsuitable complications these as the failure to discover global optimisation value, low convergent speediness and so on. P. D. Sathya et al. [46] suggested bacterial foraging established optimal multilevel thresholding, encouraged by the foraging habits of the E. coli microorganisms available in human intestinal tract. BF procedure is basic and direct and it supplies excellent efficiency based upon the eminence of the service and the speediness of the convergent than the other prevailing multilevel thresholding techniques. The effectiveness of the $\mathrm{BF}$ procedure relies on the chemo taxis step size; big action size assists microorganisms to discover optimal location much faster however not make sure the global optimum. On the other hand little chemo taxis step size warranties that microorganisms will discover global optimal however it needs huge running time. A couple of cutting edge approaches have actually been stated in the research for multilevel thresholding based upon bio-inspired processing standard such as upgraded Bat Procedure Alihodzic et al. [2], Honey Bee Breeding optimisation Horng et al. [23], Artificial Bee Nest procedure Horng et al. [24] and Firefly Procedure (MTF) Liou et al. [25]. Uroš Mlakar et al. [44] suggested an ideal multilevel image thresholding utilizing a hybrid differential development method. Multilevel image thresholding utilizing honey-bee-mating approach has actually been suggested by Yunzhi et al. [32]. In 2017, Nipotepat et al. [45] suggested a multilevel thresholding strategy utilizing differential development procedure. Just recently, a bio-inspired procedure for multilevel image thresholding based upon whale optimisation procedure and moth-flame optimisation has been recommended by Mohamed Abd El Aziz et al. [11]. The above techniques carry out sufficiently; accomplishing finest values for the unbiased function, the computing expense sustained is high.

\section{Literature survey}

FCM is a prominent, center-based clustering strategy because of its simpleness and effectiveness with narrow intricacy. The option of the FCM procedure depends on the preliminary arbitrary state and consistently converged to the regional. Just recently, scientists to conquering 
this issue have offered heuristic clustering procedures. Because of the huge quantity of info along with the intricacy of the problems, classic optimization approaches are unable of resolving a lot of the optimization problems; consequently, scientists have begun to utilize meta-framework procedures. Today, nature-inspired procedures are extensively made use of to resolve these problems in numerous areas $[12,13,48]$. Clustering strategies and various other information mining and information evaluation actions have made considerable development making use of the cumulative intellect procedures. Clustering with heuristic procedures is becoming an option to even further traditional clustering strategies $[8,62]$.

The researchers have really advised various hybrid methods for image segregation. Christe et al. [3] made the adaptation in among K-means along with fuzzy C-means. They picked the selection of clusters, fuzziness, variety, in addition to quiting the need. They triggered the member randomly or getting from K-means designs, recalculating centers and similarly membership till the impartial attribute stretched. The superiority of their method is that it can care for overlaying grey range intensities. The downside of their suggested strategy is that it cannot obviously precise borders among cells positively. It decreases within-class sum square errors, nonetheless its effectiveness deteriorate in which utilized to noisy affected image. They fixed this difficult by the pre-processing activity formerly utilizing the consolidation. They distinguished their effect with KM, FCM, adaptation FKM in circumstances of under-segregation along with over- segregation. They confirmed that FKM offers the very least under or over- segregation, yet they did disappoint simply what involving to time of the adaptation technique.

Funmilola et al. [1] made the Fuzzy K-C-means technique, which brings a lot more of Fuzzy C-means suggests belongings contrasted to that of K-means. The procedure evaluate the images, develops the design, reduce the design by detachment, acquires the dimension of the images, with each other the dimensions, produces huge info points by range estimate, similarly reduces duplicating when viable variety has really been accomplished. The variation begins by figuring out considerable aspect of details afterwards it gives up when viable recognition ends. Fuzzy K-C-means manage grey range image like Fuzzy C-means. It yields the identical range of variations as in Fuzzy C-means. In which reduced the designs by inspection out the ranges simply. The adverse element is that the effect of their endorsed system appears like completion end results of the Fuzzy C-means process besides in certain images. The instant of Fuzzy Cmeans is more than by optimum 2's contrasted to their suggested system.

FCM process has really exposed enticing cause segregation by enlightening the density of the locations. This process is pixel-wise clustering wherever, similar pixels require continuing to be in the precise very identical group whereas, and the various ones are elected to dissimilar clusters. FCM system aids from simplicity of its implementation, it has some considerable problems. Cluster center would certainly be stimulated arbitrarily while using FCM. In this problems may have damaging effect on completion result of the segregation high quality. To dominate this concern, some scholars endorsed a selection of dependable initialisation approaches $[6,36]$. The fuzzy Maximum Likelihood Evaluation (FMLE) was employed as a clustering technique for determining details consider [6] where, FCM procedure was made use of as an initialisation main action for the FMLE procedure. In [36], a centroid initialisation strategy, called obtaining fragmented, was recommended, where every n-dimensional instances are changed to one dimensional by acquiring the mean over the feature location. All instances are regularly separated right into $\mathrm{C}$ groups along with $\mathrm{C}$ portions of indices that are repeatedly created. Cluster centers are resolved. The secondary problems pertaining to FCM is the range of clusters, the $\mathrm{C}$ value, which require to be identified previously. Countless 
trustworthiness indices have in fact been advised to identify one of the most efficient ranges of clusters [6]. Several authenticity indices have in fact generated numerous cluster numbers that rely on the group of the precise details. The aspect is that a great deal of these advised techniques are based upon logical information, along with they are generally conscious texture information, like real images, which might have appearance and lightning.

Mualik and also Mukhopadhyay [41] offered a consolidated clustering procedure. They incorporated SA (Simulated Annealing) with ANN (Artificial Neural Networks) to enhance the resulting performance. The recommended hybrid procedure was made use of cluster three realistic microarray information along with the outcomes of suggested techniques were related to some typically utilized clustering procedures. The outcomes suggested the prevalence of the new procedure.

An excellent numerous durable meta-heuristic searching techniques that are motivated naturally have been developed to address difficult engineering problems [55], like system recognition [22], criterion evaluation [39], engineering optimization [18, 58], and educational [10]. An extensive number of meta-heuristic techniques can constantly locate optimum or suboptimum solutions from a populace of solutions. In the past 20 years, numerous popular optimization methods have been established, like ant colony optimization (ACO) [28], genetic programming (GP) [16], particle swarm optimization (PSO) [52] and also a lot more just newly, the KH procedure [17] that is based upon the simulated of the swarm habits of krill.

In 2012, a swarm intellect method, specifically KH technique [17], was first of all provided for the global optimizing issue. The $\mathrm{KH}$ method attracts its example from the herding actions of krill in nature. The detached feature utilized in $\mathrm{KH}$ technique is primarily determined by the minimum ranges of the location of the sustenance and also the highest swarm denseness. The location for every krill primarily conceals three components.

$\mathrm{KH}$ is a reliable technique in exploitation. On event, it might not run away several regional finest options in multi-model fitness landscape so that it cannot look around the global [41]. For routine KH technique, the search depends completely on randomness; as a result, it cannot precisely converge immediately. EKHA was realized to be sufficient affordable with various other widely known and prominent meta-heuristic techniques.

\subsection{Problem declaration}

In video security, identification of moving objects from a video is vital for object identification, target tracing and activity understanding. Area of moving objects in videos is the primary essential endeavor of information and back-ground reduction is a popular approach for foreground segregation. In this inference, we have recreated distinct background reduction methods to overcome the problem of lightening range, back-ground mess and shades. Back-ground and also leading edge of body positions is crucial in recognizing human workouts. Smart and robotized protection monitoring structures have developed into a working expedition area in on-going time as a result of an increasing rate of interest for such structures visible areas, for instance, air terminals, underground terminals and mass events. In this particular situation, the tracing with stationary foreground location is among one of the most standard requirements for security structures depending on the tracing of abandon or stolen objects or left vehicles. Object tracing and also discovery based approaches are one of the utmost popular choice to identify motionless closer view objects given that they function smartly well when the video camera is motionless and the transformation in including lightning is continual, and also furthermore talk to one of the most common choice to separate closer view objects from the current edge. 
Object tracing and discovery is a basic issue either it is used in lots of real-world process that is criminal crime discovery, motion identification, and video security and so on. The intricacy of the issue relies on the kind of object and atmosphere we are selecting. Still, there are a lot of complications offered in object tracing and detection [30, 40, 43, 57]. In our suggested technique, we are making use of objects with occlusion which is one of the essential subjects in object detection.

1) The function of multi-object tracking is in inferring undertakings when it comes to details objects from a video collection, which thought about as a dynamic detailed spatiotemporal clustering problem.

2) The forefront mining techniques are likewise not appropriate for identifying moving objects in video taken through moving cameras.

3) Self-occlusion, inter-object occlusion, along with occlusion with help of background scenery structures are the three classifications of concerns in the object tracking procedure.

\subsection{Objectives}

- Recognition of interesting motion objects.

- Tracking regarding these objects beginning from all frames.

- Assessment of object tracking to determine their motions.

\section{Proposed system model}

Object tracing is a dynamic strategy since the temporal details connecting with the previous frame structures. Signifying an approach with much more prominent accuracy in complicated scenery formations is challenges for researchers. In this exploration study, involving objects location to that activity tracing video is advised. The recommended technique has few remarkable tasks, for example, background and forefronts location in addition to tracks moving objects. Beneath background along with forefront location is finished by hybridization of SKH-RKFCM clustering system. Last task is tracing moving objects; at first the characteristics are drawn out from the segregated frame structures whereby objects forefront is determined as blob. In Fig. 1 the fundamental structure is exposed. The representation of recommended approach is exposed below.

The video configuration is directly appropriate into ' $N$ ' selection of frame structures. The 'N' variants of frame structures are prompted to the background and forefront segregation. In background along with foreground segregation, SKHA-RKFCM clustering procedure is recommended. In which the background along with foreground segregations are done, then the object tracing is complete from frame structure to arrangement taking advantage of where objects forefront is determined as blob. Above stages of upcoming arrangement is revealed up in under,

1. Back-ground and foreground partition.

2. Trace the moving objects. 


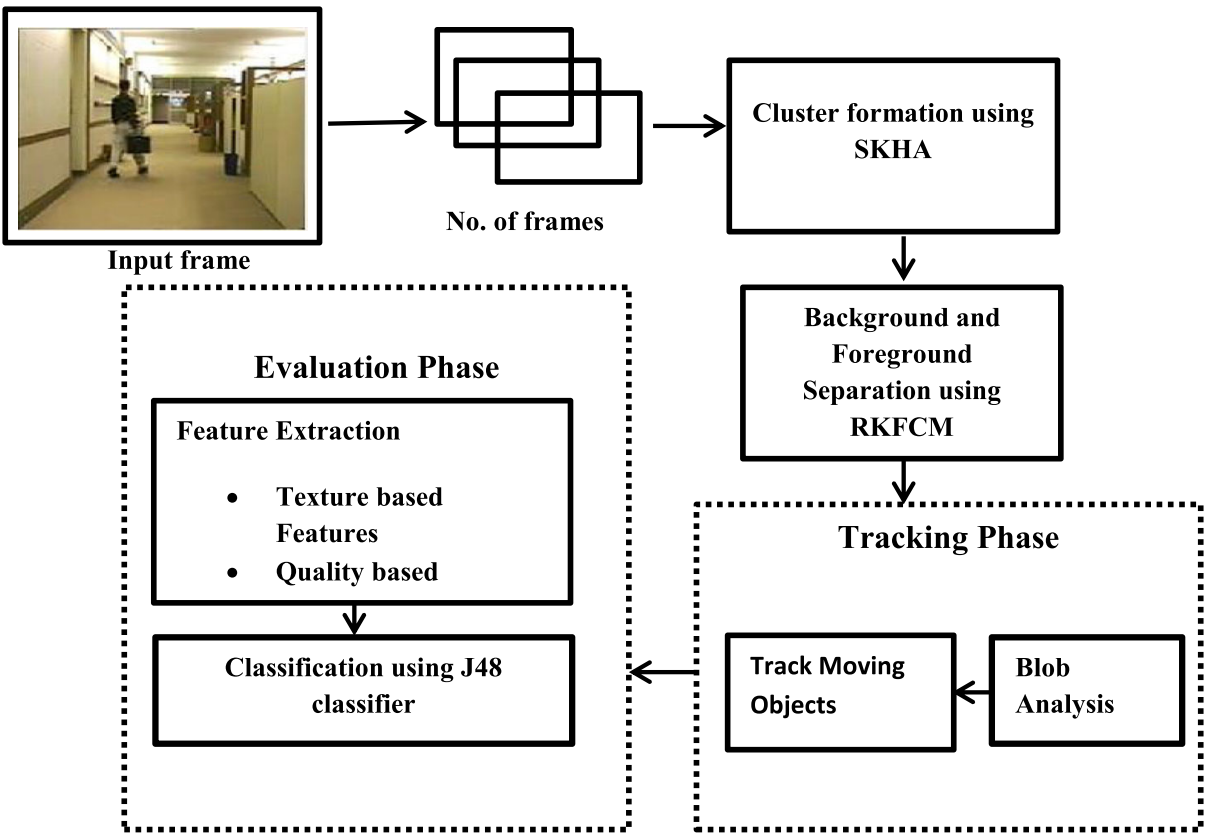

Fig. 1 The proposed system model

\subsection{Segregation of background and foreground}

The strategy of getting moving forefront objects commencing conserved back-ground image or through back-ground estimation from image gathering is called back-ground devaluation. In among one of the video reductions, the objects of complication remain in the forefront of a section wherever aspects are occurring and also movements are happening. Bearing in mind conclusion purpose to trace the advancement of these objects, it is asked for to leave along with recognize them starting the settled background before either kind of sort of extra handling. The intensity of the pixels recommending to the exercised back-ground takes place being greatly unchanged in the center of two active frame structures. By identifying the distinction, these pixels get well stabilized along with simply moving forefront location objects are conserved. In background and foreground procedure finished by SKHA clustering procedure comprised with Refined Kernel Fuzzy C-Means (RKFCM). The complete approach of SKHA together with Refined Kernel Fuzzy C-Means (RKFCM) clustering system is clarified in below.

\subsection{Overview of swarm intelligence}

Swarm intelligence methodology, particularly KH methodology [17], was at first provided for the global optimizing issue. The $\mathrm{KH}$ technique attracts its similarity starting the herding practices of krill in natural surroundings. The intention functionality made use of in $\mathrm{KH}$ technique is mainly determined through the least ranges of the location of the sustenance and the highest swarm denseness. The location of every one krill mainly addresses three locations. 
$\mathrm{KH}$ is a reliable approach in exploitation. On event, it might not discharge certain regional finest solution in multimodal suitability scenery so that it cannot examine globally well [41]. For systematic KH technique, the examine depend on totally on arbitrariness; for that reason, it cannot continuously congregate quickly.

In basic GA (hereditary algorithm) [20, 21], some hereditary operatives (selection, crossover and anomaly) reoccurrence till a conclusion scenario is fulfilled. To increase the effectiveness of GA, a series of hereditary algorithm has been established. Among the very popular techniques is Stud GA (SGA) [33] In SGA, instead of stochastic choice, the best individual, the Stud, offers its valuable info for all the further individuals in the populace by GA operatives [33].

Within this study, a reliable SKH approach merging KH with SGA is recommended. The goal of SKH is to speed up confluence rapidity. Now the first phase of SKH, we make use of standard $\mathrm{KH}$ to select an ideal attractive result setting. Consequently, for more precise design of the krill habits, stimulated by SGA, an efficient selection and crossover procedure, termed stud selection and crossover (SSC) operative, is contributed to the technique. The SSC operative is used to tweak the picked favourable resolution in order to boost its dependability and effectiveness for global optimizing. The included SSC operative rationalized the krill's location conferring to the roulette wheel selection. The crossover process in SSC operative can assist to prevent precipitate confluence in the primary run phase, and enhance the last options in the later. Empirical outcomes specify that SKH completes more effectively and robust than the $\mathrm{KH}$, and further optimizing techniques.

\subsection{KH algorithm}

$\mathrm{KH}$ [17] is a new universal stochastic optimizing technique for the global optimizing problems. It is influenced through the krill swarm while searching for the food and interacting with all various other. The KH technique restates the execution of the three activities together with adheres to hunt paths that expand the objective feature value. The moment depend location is mainly established by three activities.

- foraging activity;

- Motion affected by various further krill;

- Physical diffusion.

Normal KH method embraced the Lagrangian version [17] as received the adhering to declaration:

$$
\frac{d X_{i}}{d t}=M_{i}+F A_{i}+P D_{i}
$$

In which $F A_{i}, M_{i}$, and $P D_{i}$ represent the foraging movement, the activity influenced by various alternative krill, the physical diffusion of the krill $i$, specifically. The very beginning activity $F A_{i}$ dealt with two sections: the present food position along with the details regarding the previous position. With regard to the krill $i$, we developed this activity below:

$$
F A_{i}=V_{f} \beta_{i}+\omega_{j} F A_{i}^{\text {old }}
$$


Where

$$
\beta_{i}=\beta_{i}^{\text {food }}+\beta_{i}^{\text {best }}
$$

Along with $V_{f}$ is the foraging rate of speed, $\omega_{j}$ is the inactivity weight of the foraging activity in $(0,1) F A_{i}^{\text {old }}$ is the recent foraging activity.

The path recognized by the second motion $M_{i}, \alpha i$, is approximated by the three impacts: target result, regional result, and also revolting impact. Since a krill $i$, it can be developed beneath:

$$
M_{i}^{\text {new }}=M^{\max } \alpha_{i}+\omega_{n} M_{i}^{\text {old }}
$$

In which $M^{\max }$ is the optimum stimulated rate of speed, $\omega_{n}$ is the inactivity weight of the second progress in $(0,1) M_{i}^{\text {old }}$ is the recent progress affected by various other krill.

Regarding $i^{\text {th }}$ krill, in fact, the physical diffusion is an arbitrary procedure. This movement consists of two sections: an optimum diffusion rate of speed and orientated vector. The declaration of physical diffusion can be offered below

$$
P D_{i}=P D^{\max } \delta
$$

In which $P D^{\max }$ is the optimum diffusion rate of rapidity, and $\delta$ is the orientated vector whose values are arbitrary quantities among - 1 and 1 .

Based on the three above-evaluated activities, the time-relied location since time $\mathrm{t}$ to $t+\Delta t$ can be developed through the equation:

$$
X_{i}(t+\Delta t)=X_{i}(t)+\Delta t \frac{d X_{i}}{d t}
$$

Most significantly, keep in mind that $\Delta t$ is a vital criterion together with needs to be managed in regard to the specific real-life issue. The factor is that, somewhat, this criterion can be dealt with as a range element of the rate of speed along with includes the variants of the global finest interest, and its value is of crucial relevance in establishing the rate of the convergent and how the $\mathrm{KH}$ operates. Even more information concerning normal $\mathrm{KH}$ method the three primary steps can be denoted as $[17,55]$.

\subsection{SGA (stud GA) and genetic algorithm}

GA and SGA are established straightforward to GA, as a result first of all a quick summary of GA is presented in this segment.

Genetic algorithm (GA) GA is an authoritative stochastic meta-heuristic exploration approach for the global optimisation in a huge exploration area. The genetic info is inscribed as genome that is applied in an unusual manner in which allows a sex-related recreation that results in the spawn that are hereditarily the very similar by the parent. Whereas sex-related recreation can swap and reorder chromosomes, bring to life spawn that contain incorporation of hereditary info from all parents. This procedure is often termed crossover due to the fact that the chromosomes crossover while switching hereditary info. To escape early convergent, mutation is used to enhance the variety of the populace. A basic GA process has the adhering to steps: arbitrarily activating a populace of contestant results, producing new children by hereditary controllers. The fitness of the recently produced results is about computed and well-fitted 
selection system is after that made use of to determine which results will certainly be kept in to the future generation. This procedure is after that replicated till a predetermined variety of generations is achieved or some quit standard is completely fulfilled.

Genetic procedures have been extensively made use of given that it is established, and GA has confirmed to prosper in resolving numerous standard practical engineering complications.

SGA (stud GA) A SGA [33] is a kind of GA that uses the optimum genome for crossover at all generation. The concept of SGA is to utilize the optimum genome to mate with all others to produce new spawn [33]. Right here, SGA do not utilize stochastic selection. The SGA can be provided as adheres to [49]:

Stage1. Activate a populace randomly.

Stage2. Select the optimum genome for mating.

Stage3. Execute crossover.

Stage4. Repeating till terminating standards is fulfilled.

The crossover procedure is the very important of the SGA. As a whole, the SGA applies in regard to the adhering to actions.

- Disarrange two stud features (choose arbitrarily).

- Examine the diversification depending on the hamming proximity among the disarrange stud and the present mate:

- If diversification is larger than an established threshold, execute crossover to produce one spawn.

- Else, mutate the present mate to produce the children [49]

- Repeating for all various other mates [33].

\subsection{SKH}

Due to the fact that the exploration utilized in the routine $\mathrm{KH}$ approach counts entirely on arbitrariness, the $\mathrm{KH}$ cannot constantly locate the optimum results. In $\mathrm{KH}$ procedure, adaptive hereditary recreation systems have been presented so regarding enhances its efficiency [17]. On event, KH might not be effective in progressing on far enhanced results on certain spatial challenging problems. Normally, the standard $\mathrm{KH}$ approach is expert at examining the searching area broadly along with finding the position of global finest results, yet it is unacceptable at determining results in a greedy way [55]. In today research, inter to significantly progress the efficiency of $\mathrm{KH}$, comparable to adaptive genetic recreation systems [17], an upgraded genetic recreation systems, termed stud selection and crossover (SSC) controller, is presented in to the $\mathrm{KH}$ technique to establish a SKH method to maximize the baseline features. The presented SSC controller is motivated through the distinguished SGA. Right here the feature of all-natural advancement is rendered with the initial krill to bring to life a version of tremendous krill that is well with the ability of applying the SSC controller. In SKH, the SSC controller is used to just take control of the freshly producing far superior results for each 
and every krill; whereas in $\mathrm{KH}$, it is prone to approve all the upgraded krill. The structure of SSC controller is presented below.

Algorithm1: Selection and crossover (SC) controllers

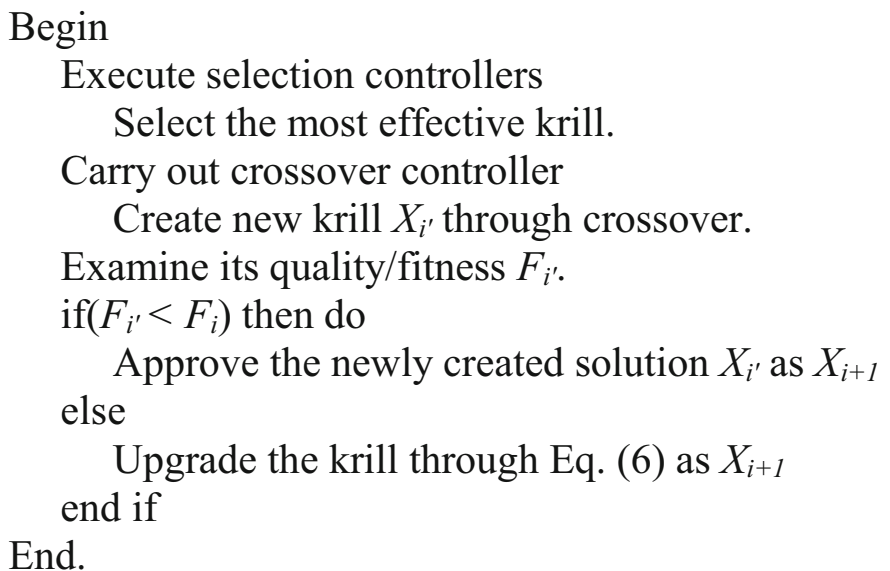

From Algorithm1, we can observe that SSC controller really deals with two small controllers: selection and crossover. Comparable to SGA, the suggestion of the SSC controller is to utilize the optimum krill (the Stud) to mate with all the various other krill to produce the child krill instead of it is not perfect results. In significance no stochastic selection is made use of in SSC controller.

Within Algorithm1, to start with, the Stud, i.e., the optimum krill is selected as the initial parent. And after that, an additional parental is chosen to mate with the stud develop two offspring by roulette wheel selection. Keep in mind that we should see to it the research study is not picked as the 2nd parent. Next off, making use of two parents, and unique krill $X_{i}$, is produced by some type of crossover (Crossover controller). The crossover procedure is the heart of SSC controller. The high quality of the freshly produced spawn $X_{i^{\prime}}\left(F_{i^{\prime}}\right)$ is approximated by the objective feature. If $F_{i},<F_{i}$ we approve this freshly produced krill $X_{i}$, as $X_{i+1}$ in the future generation. Or else, the moment relevant location of the krill in the exploration area is upgraded by Eq. (6) as new result $X_{i+1}$ in the future generation. This is so relatively greedy selection technique that it can permit the entire populace continue to far enhance results and not get worse the populace constantly.

Within SKH, to start with, the normal KH strategy is related to decrease the searching location to a much more encouraging location. Where after, the uniqueness of SSC controller that is an excellent greedy approach is made use of to just take control of enhanced results to much superior the high quality of the results. Via in this manner, the suggested SKH method can explore the entire area broadly by fundamental $\mathrm{KH}$ technique and extraction of helpful details by SSC controller. Each excellent expedition of the standard $\mathrm{KH}$ approach and the phenomenal exploitation capacity of the SSC controller can be totally applied. In fact, based upon the form of SKH, the normal KH in SKH stresses the global exploration at the beginning of the procedure to leave from regional results; whereas consequently SSC controller motivates the regional searching in the exploration area at the later run stage of the procedure. Via this reliable system, the recommended SKH technique can take complete usage of the substantial expedition of the $\mathrm{KH}$ and struggle with the weak regional searching of the standard $\mathrm{KH}$ 
technique. Reviewing to various other optimization techniques, this might be a benefit for this technique as we can see in the simulations below. Most significantly, this approach cans even more clearly the major dispute in between expedition along with exploitation successfully. By combining above evaluated SSC controller along with standard KH approach, the SKH has been established, and also the structure of the SKH can be defined in Algorithm2. Below, $N P$ is the dimension of the parental populace $P$.

Algorithm 2. SKH procedure

Begin

Stage 1: Initiation. Establish the Genesis Counter $\mathrm{t}=1$;

Activate the populace $P$ of $N P$ krill; established the foraging rate of rapidity $V_{f}$, the

Optimum diffusion rate of rapidity Dmax, and the optimum stimulated rate of speed

Nmax; a probability of crossover pc.

Stage 2: Assessing populace. Review the krill populace based upon its location.

Stage 3: While to MaxGenesis do

Arrange for every krill depending on to their fitness.

for $\mathrm{i}=1$ : NP (all krill) do

Execute the three movements.

Upgrade location for krill I by SC controller in Algorithm 1.

Assess for each and every krill depending on its new location $X_{i+1}$. end for $\mathrm{i}$

Arrange for every krill and locate the present best.

$t=t+1$;

Stage 4: end while

Stage 5: Compute the most effective results. End.

\subsubsection{Clustering based on SKH}

Clustering remains in conformity with suitable indexes to locate an optimum clustering procedure. The significance of clustering is the optimization procedure. What is very essential is locating methods to incorporate the optimization procedure SKH with clustering. By standing for every krill (each pixel) in the SKH as a clustering system, we locate the optimum clustering plan by selecting the suitable objective functionality. A clustering plan can be revealed by all clustering centers. That is, every krill ' $X_{i}$ ' stands for the ' $K$ ' cluster centres:

$$
\begin{gathered}
X_{i}=\left\{C_{1}, C_{2}, C_{3}, \ldots \ldots C_{k}, \ldots \ldots C_{k-1}, \ldots \ldots C_{k}\right\} \\
C_{k}=\left\{C_{k}^{1}, C_{k}^{2},, C_{k}^{3}, \ldots \ldots C_{k}^{d}, \ldots \ldots . C_{k}^{D-1}, \ldots \ldots \ldots \ldots C_{k}^{D}\right\}
\end{gathered}
$$


Where 'd' would certainly' signifies the number of criteria of the information that will certainly be clustered, and also ' $C_{k}^{1}$ ' stands for the very first criterion of the initial cluster center. Each krill individual can be revealed as the complying with matrix (row column values).

In this research revision, one krill is utilized to stand for a prospect solution to an issue, and also the picked ' $K$ ' first cluster centers are possible options. One krill and ' $K$ ' preliminary clustering centre play comparable functions in our procedure. Hence, the mapping in among a krill and ' $K$ ' first clustering centers can be developed. In the coding approach of the krill location framework, a collection of first cluster centres are produced arbitrarily from the dataset factors.

The entire krill populace stands for a range of clustering systems. In this fashion, our objective is to locate the optimum clustering centres. Depending on the concept of the marginal range, information is classified right into the suitable cluster. The summary of the stud krill-herd clustering procedure is received in procedure 1.

\subsection{2 procedure1. Stud krill herd clustering procedure}

(1) Specify the criteria $\left(K, I_{\max }, N, N_{\max }, V_{f}, D_{\max }\right.$, and more).

(2) Activate $\mathrm{N}$ krill arbitrarily as the first clustering centre.

(3) Assess every krill by fitness feature.

(4) For every krill: Carry out three movements (movement stimulated by one more individual, foraging movement and physical diffusion).

- Execute the crossover operator and the enhanced mutation operator (two mutation systems were carried out for individuals with various fitness degrees).

- Compute the fitness conferring the krill's new location; if the novel fitness is far superior to the older, upgrade the krill individual location in the exploration area.

(5) Make usage of the upgraded system to upgrade the krill's location if the novel location transcends to the prior location.

(6) Recurrence order 4 and 5 till the quiting standards are completely fulfilled.

(7) Go back to the most effective clustering solution.

\subsubsection{Fusion of SKHA}

The significant objective of this part was to reveal the mathematical activities related to the details clustering utilizing the optimal centroid developed making use of the recommended SKH procedure produced by including a special fitness attribute with the recommended procedure. The optimum centroid is used to arrange the details variables right into clusters remembering the entire objective functions. While clustering, primarily, the info variables and the clusters are activated in order to develop the optimal value of the centroid. The advised SKH system explores for one of the most efficient centroid for implementing the info clustering. The SKH process for determining the optimal centroid along with and also centroids are estimated optimum based upon the minimize value of the fitness action. The fitness value relies on three restrictions, specifically the intra-distance, interdistance and the denseness of the clusters. The optimal centroid developed utilizing the recommended SKH procedure is used cluster the details elements. For execution the details clustering, the 
least distance among the clusters along with the info variable is developed the centroids matching the details variables of the least distance are arranged with every various other in a solitary group.

\subsection{Refined kernel fuzzy C-means (RKFCM)}

Refined kernel fuzzy c-means process is encircling the kernel fuzzy c-means process through several kernels learning system. The objective function of refined kernel fuzzy c means process is well referred to as follows.

$$
O B(F, C)=\sum_{i=1}^{N} \sum_{j=1}^{c} M_{i j}^{m}\left(1-K_{M K}\left(F_{j}, C_{i}\right)\right)
$$

Wherever,

$\mathrm{M}_{\mathrm{ij}} \quad$ indicates membership of $\mathrm{j}^{\text {th }}$ data in the $\mathrm{i}^{\text {th }}$ cluster $\mathrm{C}_{\mathrm{i}}$

$\mathrm{F} \quad$ indicates input frame

$\mathrm{C}$ indicates midpoint of cluster

$\mathrm{K}_{\mathrm{MK}}$ indicates multiple kernel function

\section{Phases for RKFCM}

phase 1: initializing the frame (F), amount of cluster $(\mathrm{C})$ and amount of kernels $(\mathrm{K})$.

phase 2: initializing the association matrix $\mathrm{M}$.

phase 3: The centre of the cluster is estimated employing the succeeding Eq. (10),

$$
C_{j}=\frac{\sum_{i=1}^{N} M_{i j}^{m} F_{i}}{\sum_{i=1}^{N} M_{i j}}
$$

phase 4: upgrade the association function succeeding the Eq. (11),

$$
M_{i j}=\frac{1}{\sum_{K=1}^{C}\left(\frac{\left\|F_{i}-C_{j}\right\|}{\left\|F_{i}-C_{K}\right\|}\right)^{\frac{2}{m-1}}}
$$

$\mathrm{m}$ is whichever real number $>1$.

Now refined kernel fuzzy c means, we are taking into factor to consider of various kernels as our proposed design. So $\mathrm{k}_{\mathrm{MK}}(\mathrm{a}, \mathrm{b})=\mathrm{k} 1(\mathrm{a}, \mathrm{b})+\mathrm{k} 2(\mathrm{a}, \mathrm{b})$ is a kernel.

$$
K_{M K}(a, b)=K_{1}(a, b)+K_{2}(a, b)
$$




$$
\begin{gathered}
K_{1}(a, b)=a^{T} b+c \\
K_{2}(a, b)=1-\frac{\|a-b\|^{2}}{\|a-b\|^{2}+c}
\end{gathered}
$$

C indicates always constant.

Since the aforementioned equation, centre of the cluster Eq. (10) and association Eq. (11) is reformed. Instantly centre of the cluster calculation is carried out through Eq. (15)

$$
C_{j}=\frac{\sum_{i=1}^{N} M_{i j}^{m} k_{M K}(a, b)}{\sum_{i=1}^{N} M_{i j}}
$$

In consuming the Eq. (16) the association is upgraded,

$$
M_{i j}=\frac{1}{\sum_{K=1}^{C}\left(\frac{\left\|K_{M K}(a, b)-C_{j}\right\|}{\left\|K_{M K}(a, b)-C_{K}\right\|}\right)^{\frac{2}{m-1}}}
$$

phase 5: if $\left\|M^{(K+1)}-M^{(K)}\right\|<T$, if the condition is satisfied terminate, else forward to phase 3.

According to the RKFCM the foreground and background objects are separated. Afterward the foreground and background isolating, the subsequent outcome is served to the tracking process.

\subsection{Suggested SKH -RKFCM algorithm}

Using SKH algorithm associated to RKFCM does fuzzy cluster. One facet is the efficacy of SKH process can assist the discrimination of RKFCM initialization. On the different other hands, the parallel along with distributed computing of SKH process identifies optimal centroid and increases clustering efficacy. It is important that knowledgeable exploration and self-adapting might help obtain global optimal. RKFCM clustering is between fuzzy clustering processes and might offer level of degree of association of every image.

\section{Algorithm:4 SKH-RKFCM Procedure}

Step1: To obtain the optimal cluster centroid initiation SKH clustering process.

Step2: Establish association matrix M.

Step3: Cluster centre is calculated using Eq. (10),

Step4: upgrade the association function employing the earlier stated Eqs. (11), (12), (13), (14).

Aforementioned equalization the cluster centre equalization (10) and membership equalization (12) is customized. Currently the cluster centre estimation 
is finished by equalization (16).

Member updation is done by equation (16),

Step5: if $\left\|M^{(K+1)}-M^{(K)}\right\|<T$ then termination, or else forward to step 3.

Hybridization of RKFCM with SKH the foreground and background objects are effectively determined. The RKFCM procedures deals with local optimal concerns in order to get rid of the cluster based initiation issue; we carried out the robust SKH optimizing principle which assistances to supply appropriate quantity of clusters along with centre of cluster. This assists to recognize significant and effective background and foreground tracing objects.

Developed the SKH - RKFCM the foreground and background objects are segregated. Subsequently the background and foreground partition, the resulting outcome is served to the tracing process.

\section{Tracking phase}

\subsection{Blob assessment}

On computer system, visual scene blob exploration is explored as visional components were prepared for at determining elements, in addition to the image occupancies, are extremely in their lightning or shades amongst the encircling. The blob detectors have two key phases with various techniques inning conformity with its obtained expressions along with landscape intensities. Based on the modern strategy, these controllers have defined the rate of interest variable controllers, or furthermore interest rate area controllers. There is a great deal of ideas in improvise the blob along with analyzing detector. The key element will definitely use the equivalent info of the area which is not obtained through edge detectors. At a basic region the blob exploration abstractors the area of a rate of interest rapidly for even more procedure. The occurrence of the object is signalized in the area in addition to objects do away with application image domain is traced. On numerous other domains like histogram examination, blob descriptors are sought peak exploration which offers for segregation. Among one of the most regular advantages of blob descriptors is appearance analysis and appearance identification. Present times these blob descriptors have in fact ended up being added famous mostly for its big common stereo matching and showing the instructive image characteristics which are much more vital for appearance-based objects identification based upon regional image information. It is also effectual in spotted ridge exploration to signify the occurrence of prolonged objects. The overall flow diagram is shown in Fig. 2.

\subsection{Assessment stage}

\subsubsection{Texture-based abstraction utilizing revised regional self-similarity descriptor}

Regional self-similarity description captures inner geometries layout of native selfsimilarities throughout imaginings however standing for smaller area affiance deformity. The situation obtaining self-similarity of shadows, edge, replicated shapes and elaborated frameworks in a solitary unified technique. A distinct location in one image might be matches with an unvaryingly shaded location in the different other 


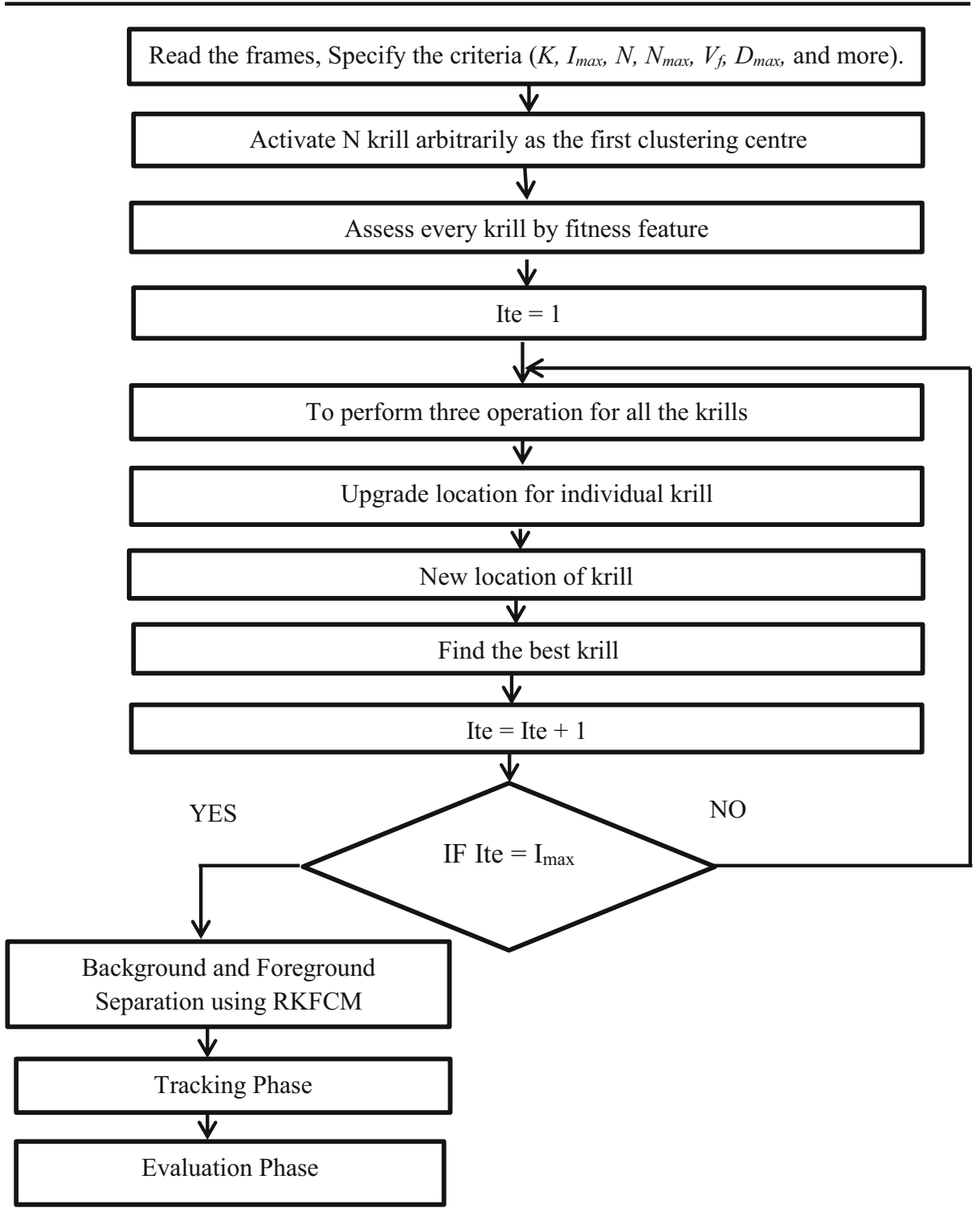

Fig. 2 System flow diagram

images as long as they have an identical geographical outline. The above mentioned self-similarity descriptions are assessed on a denseness grid of aspects image details, at many ranges. An excellent fit in among a collection of images stands for uncovering a matching collection of such descriptions with identical description values at similar geometrical setups, as long as little variable deformity. Beneath the normal regional self-similarity description is customised with the help of membership value. The in-depth summary of local self-similarity description is as shown in Fig. 3.

Derived the above procedure ourselves to discover the equivalent objects from the input video collection. 
Step 1: To locate the sum of square distinction at first acquiring an input image within the detected image $\operatorname{SSD}_{\mathrm{q}}(\mathrm{a}, \mathrm{b})$.

Step 2: To stabilize the resultant range surface area $\operatorname{SSD}_{q}(a, b)$ it is transmuted to correlation surface area $\mathrm{S}_{\mathrm{q}}(\mathrm{a}, \mathrm{b})$.

$$
\begin{aligned}
& \operatorname{Sq}(a, b)=\operatorname{Exp}\left(-\operatorname{SSD}_{\mathrm{q}}(\mathrm{a}, \mathrm{b}) / \text { Maximum }\left(\operatorname{Var}_{\text {noise }}, \operatorname{Var}_{\text {auto }}(\mathrm{q})\right)\right) \\
& \operatorname{Var}_{\text {noise }} \text { - suggests variants of image statistics } \\
& \operatorname{Var}_{\text {auto }}(\mathrm{q}) \text { - suggests framework of patterns }
\end{aligned}
$$

Step 3: $S_{q}(a, b)$ surface area of correlation is exchanged collaborates of log polar centered in $\mathrm{q}$ and subdivided right into eighty containers (angles -20, 4 radial intermissions).

Step 4: to choose the value of maxi correlation in all the containers. Because maxi container value from eighty things is our "customized self-similarity".

Step 5: Finally descriptor vector is stabilized and the range is $[0,1]$

Fig. 3 Modified procedure for local self-similarity descriptor

\subsubsection{Classification}

The last stage is the valuation stage that consists of characteristic absorption in addition to classification. The characteristic extrication is spoken about in speculative reason an extensive manner. Classification recommends identifying the images based on their qualities. Amongst that the really optimum feature is identified by three clarifiers like KNN, J48 and MLP for outcome disparities.

\subsubsection{J48 (C4.5 decision tree) procedure}

In these circumstances, we have in fact taken advantage of executing J48 decision tree approach due to the goal of classifying the video series. J48 is a unique technique for open source Java application in respect of the $\mathrm{C} 4.5$ process used currently in the WEKA details excavating technique. The situation is handed over through the task of promoting a binary tree. The decision tree approach is an unbelievable method uncovered utmost outstandingly made use of in classification problems. Using this approach, a tree is established to create the classification attribute.

\subsubsection{Overview of the C4.5 process}

- This situation is adhered to C4.5 creates decision trees specifically as of a dependable accumulation of training details with the presumption of information principle. Essentially, the training details consist of a collection of classified samples. Each sample functions as a vector which consequently it indicates functions or feature establishing component of sample.

- When it concerns all nodes either is supplied in the tree, C4.5 selects single attribute since among the details which substantially deteriorate its cluster of sample right into various subcategories improved in one kind or the different others.

- Its requirement is largely the stabilized information obtain (difference in entropy) which is triggered by selecting a features for segregating the info. The qualities with the best normalization information get are listed for the purpose of taking the decision. 
- The entire sample contained the listing are considered as individuals of the member very same kind. When it takes place, it simply creates a leaf node recommended for a decision tree with purposes to select the particular kind.

- It lies that not a scrap of the characteristics typically has a tendency to create any type of type of details obtain. Beneath, C4.5 ends up a decision node greater the tree via the excluded value of the form.

- Extremely, many conditions of a formerly undiscovered kind have really been found to take place. It is seen that C4.5 tends to develop a decision node which remains in the higher aspect of the tree using the excluded value.

- Next the framework of trees more than, it is carried out on each single tuple providing in the information resource which end up in classification for the particular tuple.

The value of the opportunity of identified objects $[P(O)]_{D T}$ in addition to the opportunity of non- $[P(N O)]_{D T}$ identified objects of decision tree classifier is figured out as of the training along with taking a look at frame dataset. Inevitably, error rate $D T_{e r r}$ and $D T_{a c c}$ also is gotten looking with the complying with mathematical declaration.

$$
\begin{gathered}
{[P(O)]_{D T}=\frac{N(D O)}{N(O)}} \\
{[P(N O)]_{D T}=\frac{N(N O)}{N(O)}} \\
D T_{\text {err }}=\frac{\text { No.of imperfectly classified objects }}{\text { Amount of objects in the dataset }} \\
D T_{\text {acc }}=\frac{\text { Completely classified objects }}{\text { Amount of objects in the dataset }}
\end{gathered}
$$

Whereas establishing a tree, J48 ignores the inappropriate valuers can be anticipated established specifically what is learnt about the particular values for the numerous dissimilar records. The principle is to splitting the details right into choice established the function values for that objects that are establish in the preparing work instance as shown in Fig. 4.

Point 1: Analyze for fundamental circumstances

Point 2: For all single attribute A, Explore the normalizing details obtain beginning with segregation regarding $\mathrm{A}$.

Point 3: Enable $A_{\text {best }}$ is the function through the very best normalizing information obtain.

Point 4: establish a decision node that separates around $A_{b e s}$.

Point 5: repeated on the sub-list obtained by separating around $A_{b e s t}$, in addition to consist of child node.

Fig. 4 J48 procedure 


\section{Results and discussion}

Our suggested structure established optimum object discovery preparing process is utilized in the performing system MATLAB. This part uses the analytical end results of the procedure carried out for clustering moving objects. These experimentations are done on an assortment of PETS, Hall monitor [9] along with various other benchmark video [57].

\subsection{Dataset explanation}

PETS Dataset: It is normally considered as wide range image evaluation in addition to have wide range count and denseness assessment, tracing of atmosphere by a wide range and discovery of different circulations and precise crowd instances.

Hall Monitor Dataset: Hall monitor trace of dataset has 300-frame in CIF resolution with slight movement. CIF images are 352 pixels wide and 288 pixels high $(352 \times 288)$.

\subsection{Effectiveness assessment}

\subsubsection{Clustering efficiency evaluation}

The strategies made use of for the comparison contain PSC, mPSC, GWO, EGWO [30], WOA [43] along with OWC [40], whose values are associated to the suggested SKH. The effectiveness assessment is achieved making use of three datasets in relation to the performance metrics along with the end results of the advised strategy are contrasted to the existing approaches. The recommended SKH surpasses the previous strategy like PSC, mPSC, GWO, EGWO, OWC, SKH (see Table 1).

Table 2 exposes the assessment of suggested SKH procedure contrasted to k-means clustering [54], Mixer model averaging [56] and OWC [40]. The analysis is utilized the PETS,

Table 1 Relative examination of clustering accuracy

\begin{tabular}{|c|c|c|c|c|c|c|c|c|}
\hline Datasets & Clustering sys & ems & & & & & & \\
\hline \multirow{5}{*}{$\begin{array}{l}\text { Dataset } 1 \text { (PETS } \\
\text { 2009) }\end{array}$} & Metrics & PSC & mPSC & GWO & EGWO(38) & WOA(39) & OWC(40) & Proposed \\
\hline & F-measure & 0.8801 & 0.6668 & 0.664 & 0.9332 & 0.9733 & 0.9821 & 0.9896 \\
\hline & $\begin{array}{l}\text { Rand } \\
\text { coefficient }\end{array}$ & 0.8697 & 0.7718 & 0.764 & 0.8678 & 0.9688 & 0.9782 & 0.9864 \\
\hline & $\begin{array}{l}\text { Jaccord } \\
\text { coefficient }\end{array}$ & 0.6712 & 0.5850 & 0.565 & 0.6684 & 0.8840 & 0.8994 & 0.9092 \\
\hline & MSE & 287.79 & 185.01 & 128.5 & 126.50 & 1.61 & 1.235 & 1.186 \\
\hline \multirow{4}{*}{$\begin{array}{l}\text { Dataset } 2 \text { (Hall } \\
\quad \text { Monitor) }\end{array}$} & F-measure & 0.4125 & 0.5398 & 0.5012 & 0.5362 & 0.8563 & 0.8864 & 0.8942 \\
\hline & $\begin{array}{l}\text { Rand } \\
\text { coefficient }\end{array}$ & 0.3312 & 0.4568 & 0.498 & 0.5067 & 0.8717 & 0.8946 & 0.8998 \\
\hline & $\begin{array}{l}\text { Jaccord } \\
\text { coefficient }\end{array}$ & 0.3368 & 0.3528 & 0.365 & 0.3656 & 0.7666 & 0.7894 & 0.7984 \\
\hline & MSE & 227,510 & 209,880 & 33,520 & 31,300 & 8027.272 & 6983.24 & 6183.56 \\
\hline \multirow{4}{*}{$\begin{array}{l}\text { Dataset } 3 \text { (Hall } \\
\quad \text { Monitor) }\end{array}$} & F-measure & 0.6584 & 0.6743 & 0.796 & 0.9312 & 0.9665 & 0.9731 & 0.9782 \\
\hline & $\begin{array}{l}\text { Rand } \\
\text { coefficient }\end{array}$ & 0.545 & 0.5456 & 0.561 & 0.603 & 0.8601 & 0.8741 & 0.8782 \\
\hline & $\begin{array}{l}\text { Jaccord } \\
\text { coefficient }\end{array}$ & 0.3552 & 0.3661 & 0.247 & 0.3661 & 0.7901 & 0.7967 & 0.7975 \\
\hline & MSE & 10,361 & 9231 & 4734 & 4734 & 760.81 & 754.32 & 743.24 \\
\hline
\end{tabular}


Table 2 Contrast through different clustering procedures

\begin{tabular}{lclcc}
\hline Metrics & K-means clustering[54] & Mixture model averaging [56] & OWC [40] & Proposed \\
\hline F-Measure & - & - & 0.9821 & 0.9896 \\
Rand coefficient & 0.645 & 0.9222 & 0.9782 & 0.9864 \\
\hline
\end{tabular}

Hall Monitor dataset for f-measure 98.96\%, 89.42\%, 97.82\% and RCs $0.9864,0.8998$ and 0.8782 as presented in Table 2 . The RC is attained in the suggested system is 0.9864 for the identical dataset, while, in k-means clustering along with Mixer model averaging, it is 0.645 along with 0.9222 . Table 2 Contrast through different clustering procedures.

\subsection{Efficiency evaluation of moving object}

The intellectual end results are presented Fig. 5 that has 6 rows in addition to 2 columns. The extremely initial 2 rows contain a structure of the video plus forefront object extracted are quit PETS video. The third and fourth rows, consisting of a structure of the video along with foreground objects obtained, are given in Hall monitor video. The fifth and sixth rows contains the structure of the video along with foreground objects are obtained in hall monitor walking video. The Fig. 6 exposes the detection along with tracked moving objects.

Within Table 3 in addition to Table 4, V1 reveals 4 frameworks, V2 reveals in 4 frames and V3 reveals in 4 frames are 3 videos in 12 frames in which Foreground (F) objects, along with detection (D) object are used. In order to analyze the effectiveness of the advised work, we abstruse some features from the fine-tuned image collection which seeks that provided to the classification. There are 2 sort of qualities are mined such as texture based attributes and quality based attributes. The logical attributes have 10 metrics are received Table 3. The texture based features contain 10 characteristics are received Table 4. These dragged out functions are transferred Attribute-Relation File Format (ARFF) also it is used to weka for a categorization. Now there three categorizer containing J48, k-nn, MLP, are used for a categorization. Each of the 3 types are accomplished in addition to its results are examined and organized.

During Table 5, the normal standards of all 3 classifiers are distinguished. Table 6 exhibits the outcome of J48, KNN and MLP categorizer particularly. The execution valuation of all 3 categorizer is displayed Table 7 wherein numerous of the requirements like Percentage of the Wrong Classification (PWC), Specificity, False Alarm Rate (FAR), Detection Rate, Accuracy, Positive Prediction (PP), Negative Prediction (NP), False Prediction Rate (FPR) and False Negative Rate (FNR) is evaluated in addition to established. With comparison, all the standards J48 provide superior execution with reduced PWC, FAR along with improved specificity, precision. Right here in Table 8, we have really distinguished our suggested J48 based object discovery strategy with previous technique [29, 40, 53] in relation to accuracy and f-measure. Our recommended system exceeds the previous strategy in Fig. 7 as an outcome of its high quality based and texture based attributes.

\subsection{Analysis}

The techniques recommended in this work are specifically concentrated to improve quality, the speediness and the functionality of the back-ground models in target 

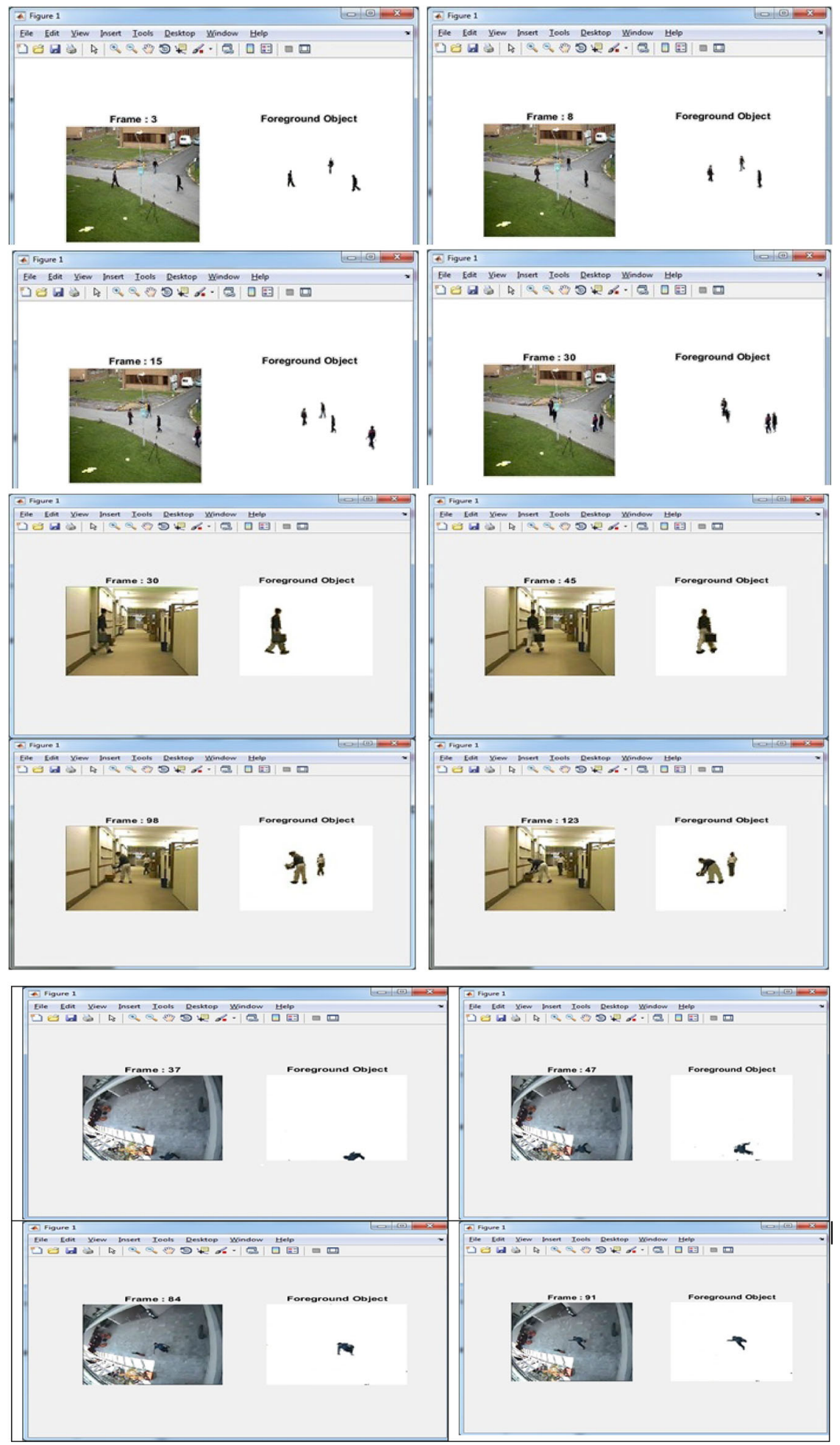

Fig. 5 Pets and hall monitor datasets shows input frame and segregation of foreground 

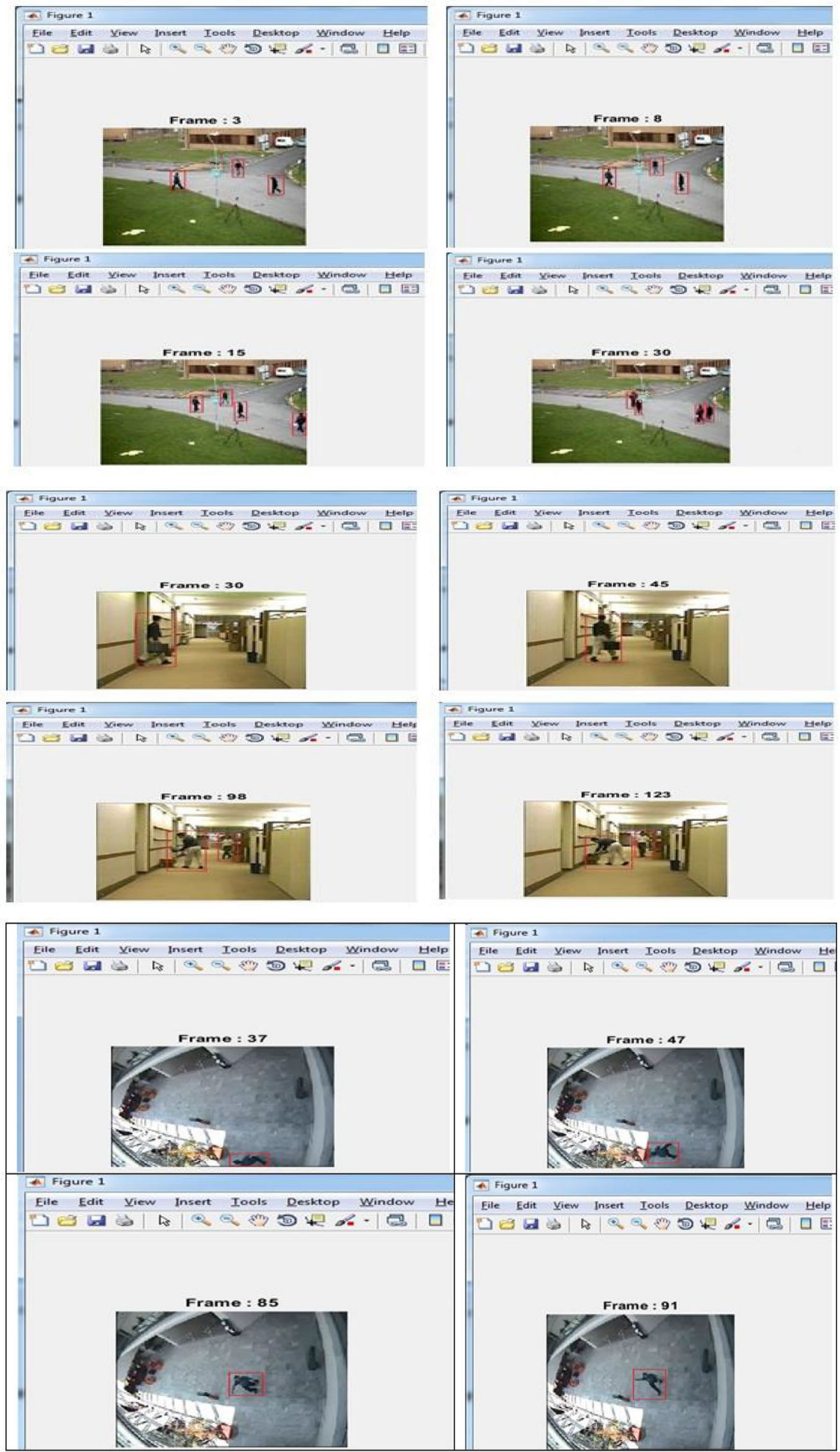

Fig. 6 Pets and hall monitor dataset results detected and tracked moving 


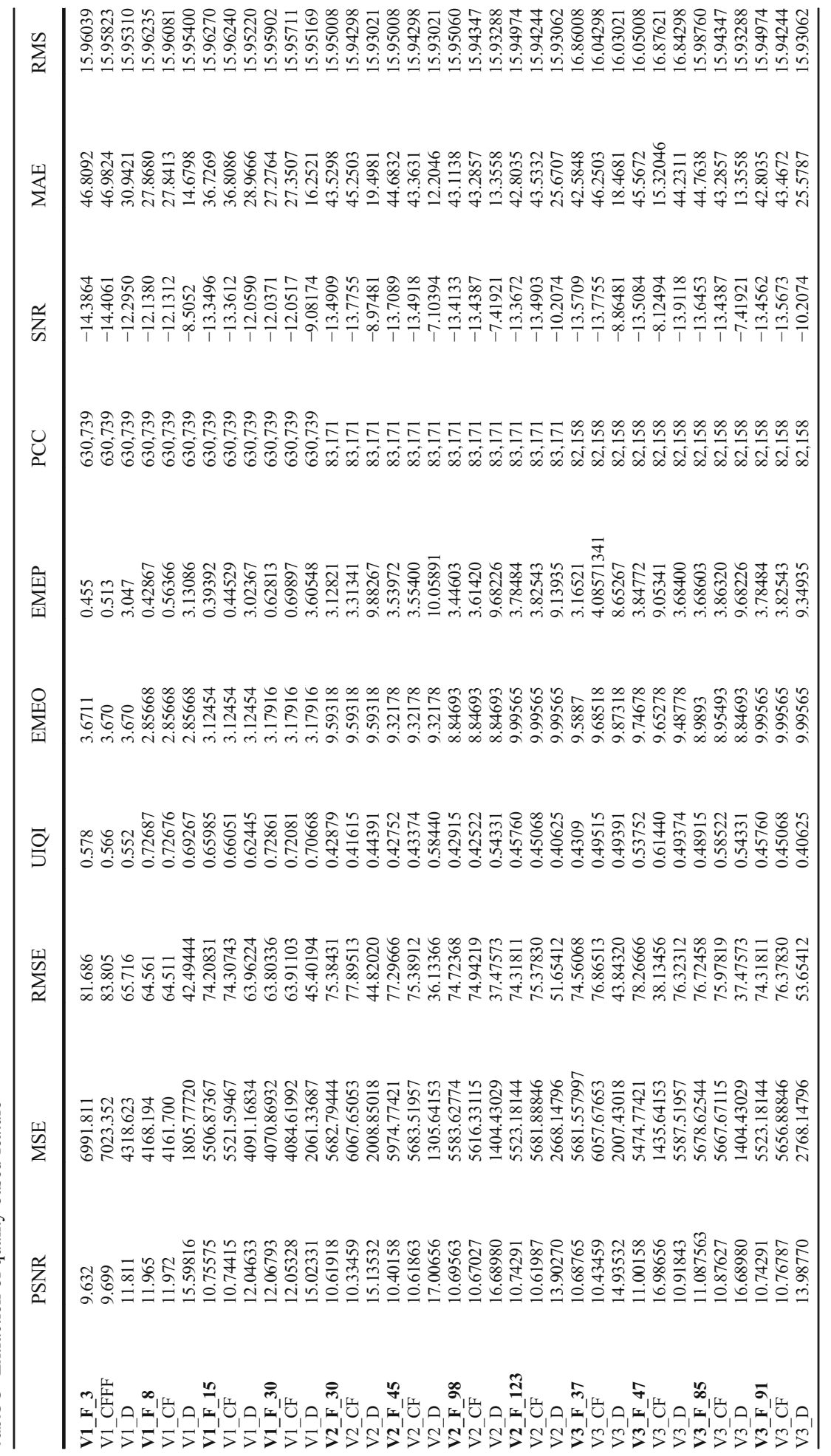




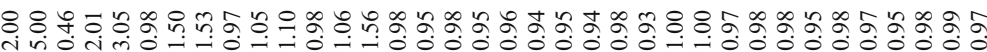

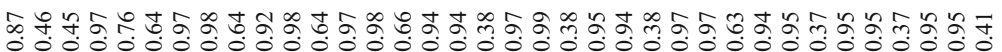

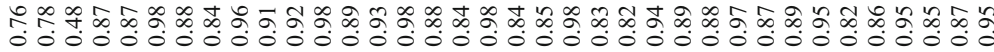

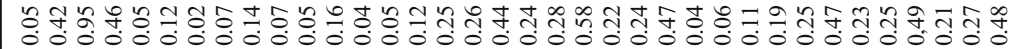

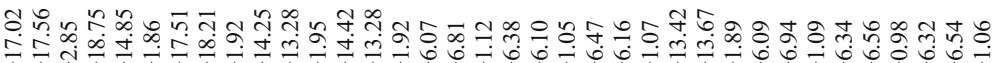

$\frac{v}{\sim}$ TTTTTTTTTTTTTYPTYTTYTTTTPYTPY PYTT

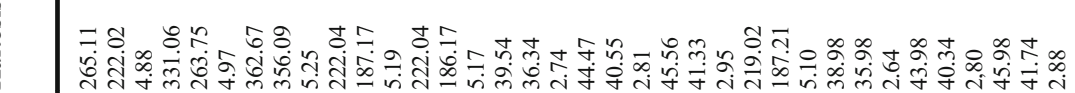

สุํํㅇำ

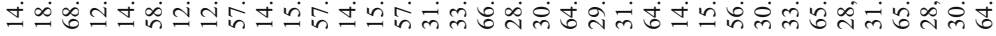

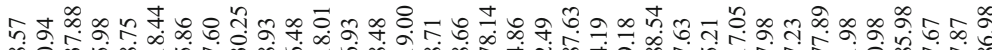

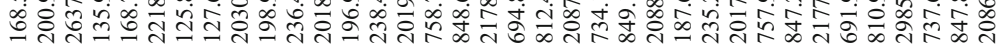

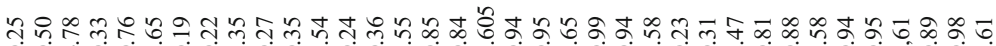
○ัण

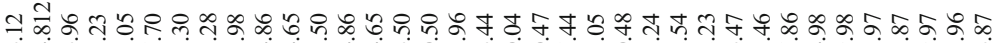

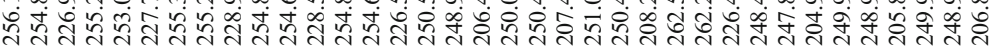

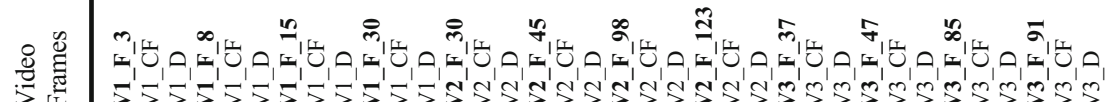


Table 5 J48, KNN, MLP evaluation

\begin{tabular}{llllllll}
\hline Classifiers & $\begin{array}{l}\text { Correctly } \\
\text { classified } \\
(\%)\end{array}$ & $\begin{array}{l}\text { Incorrectly } \\
\text { classified } \\
(\%)\end{array}$ & $\begin{array}{l}\text { Kappa } \\
\text { statistic }\end{array}$ & $\begin{array}{l}\text { Mean } \\
\text { absolute } \\
\text { error }\end{array}$ & $\begin{array}{l}\text { Root mean } \\
\text { squared error }\end{array}$ & $\begin{array}{l}\text { Relative } \\
\text { absolute error } \\
(\%)\end{array}$ & $\begin{array}{l}\text { Root relative } \\
\text { squared error } \\
(\%)\end{array}$ \\
\hline J48 & 68 & 32 & 0.328 & 0.3632 & 0.5642 & 65.9886 & 101.0312 \\
KNN & 66 & 34 & 0.312 & 0.3592 & 0.5994 & 73 & 119.4326 \\
MLP & 48 & 52 & -0.1 & 0.5821 & 0.5095 & 103.6548 & 103.9876 \\
\hline
\end{tabular}

repositioning object detection methods. In our techniques on a number of fixations outside and inside standard benchmark video collection consisting of essential circumstances such as lighting disparity, background noise, and motion variation and differing the quantity of objects in addition to object dimension. The summary of each examined video collection is as complies with:

Video footage 1 stands for more than person in the exterior scenario, and also contacting each various other. The intended object coloring is identical of the background shade of the target things is extremely similar that of the back-ground. The dimension of the frame video series is 352 pixels $\times 288$ pixels.

Video footage 2 reveals the activity of the individual from one place to alternative in opposed directives. Someone gets the box from table; all at once various other individual puts the brief-case on another table. The dimension of the frame is 240 pixels $\times 352$ pixels. This interior video has a specific degree of lighting deviations, as well as coloring of the motion objects and back-ground is identical.

In the video 3 some persons move toward from contrary directives. This video has complicated background, and it experiences lighting disparity. The frame dimension is 384 pixels $\times 288$ pixels.

\subsubsection{Qualitative analysis}

Lighting disparities Due to the considerable lighting deviations in the Video 2 and Video 3, the previous techniques deliver the imprecise outcome with more false negative and higher quantity of noise (video 2 framework138,156,162, video 3 frame 67,88,118) [14]. There are false positives and the top section of one individual in the frame 118 of Video 3 is not detected.

Table 6 Effectiveness exploration

\begin{tabular}{lllllll}
\hline Classifier & TP rate & FP rate & Precision & Recall & F-Measure & ROC area \\
\hline \multirow{2}{*}{ J48 } & 0.812 & 0.156 & 0.838 & 0.7301 & 0.7799 & 0.72 \\
& 0.750 & 0.172 & 0.813 & 0.714 & 0.760 & 0.72 \\
\multirow{2}{*}{ KNN } & 0.650 & 0.138 & 0.824 & 0.684 & 0.7473 & 0.72 \\
& 0.600 & 0.300 & 0.667 & 0.600 & 0.632 & 0.65 \\
\multirow{2}{*}{ MLP } & 0.700 & 0.400 & 0.636 & 0.707 & 0.667 & 0.65 \\
& 0.650 & 0.350 & 0.652 & 0.670 & 0.652 & 0.65 \\
& 0.000 & 0.100 & 0.000 & 0.000 & 0.000 & 0.41 \\
& 0.900 & 1.000 & 0.474 & 0.900 & 0.621 & 0.41 \\
\hline
\end{tabular}


Table 7 Efficiency examination of all three classifiers

\begin{tabular}{lllllllll}
\hline Classifier & PWC & Specificity & FAR & Accuracy & PP & NP & FPR & FNR \\
\hline J48 & 31 & 0.865 & 0.1611 & 0.798 & 0.715 & 0.616 & 0.1349 & 0.2697 \\
KNN & 45 & 0.500 & 0.334 & 0.564 & 0.637 & 0.664 & 0.501 & 0.4022 \\
MLP & 56 & 0.000 & 0.526 & 0.450 & 0.473 & 0.000 & 1.000 & 0.100 \\
\hline
\end{tabular}

Our recommended method as exposed in fig decreases the imperfection of prevailing systems for these series and also yields much more reliable and precise outcomes with not as much of false alarm system.

Disorder of background Forefront details of the object in video 2 and video 3 identical of the background. The prevailing techniques deal with the issue of forefront aperture and produce the outcomes more false negatives. Our proposed approach increases the efficiency however certain quantity of noise. The prevailing system decreases the false negatives and identified outcomes are much more accurate, however with false positives. The quantity of false negatives in the Video 2 and Video 3 is dramatically greater in this method. Our recommended technique likewise has false negatives, yet outcomes of our method are substantially a lot more precise than the previous approach [14].

Sluggish or quick movement The standard three-frame differencing and inter-frame differencing techniques (i) affected with the ghosting issues as a result of slowdown of person in Video 1 also creates the false positives. Moreover, the consequences generated by Fei et al. [14] in the Video 1 is not perfect and generate much more false positives along with false negatives. However, the recommended approach lowers the false positives in all the previously mentioned video sequences, but this strategy generates the false negative in Video 1. Thus, our proposed technique does not complete well for Video 1. Nevertheless, existing strategy outmatches all the checked techniques and also creates substantially accurate outcomes with low false positives along with false negatives for Video 1.

Deviations in quantity and dimension of objects The dimension of motion objects in the Video 3 is smaller, and differing with the object activity. The deviations of motion objects whichever video 3 contains sluggish motion. In the aforementioned video series, both the frame differencing strategies do not identify the motion objects precisely and cause the noise. The methodology suggested by Fei et al. [14] minimizes the noise in Video 3. But, it likewise produces certain false positives; thus, the motion objects are very little exact. Next, our suggested technique outmatches all the evaluated methods and discovers the objects more efficiently.

Table 8 Performance evaluations with previous approaches

\begin{tabular}{llc}
\hline Methods & Precision & F-Measure \\
\hline Proposed method & 0.825 & 0.760 \\
OWC [40] & 0.793 & 0.690 \\
Existing method [53] & 0.714 & 0.650 \\
Existing method [29] & 0.530 & 0.630 \\
\hline
\end{tabular}




\subsubsection{Quantifiable examination}

To determine the effectiveness of suggested approaches through the existing techniques, initially to define the detection error rate $\left(D E R^{i}\right)$ of all frame along with typical detection error rate $(\overline{A D E R})$ of total video utilizing the subsequent equations

$$
\begin{gathered}
D E R^{i}=\left|N_{\text {forg }}^{i}-N_{\text {ovf }}^{i}\right| / N_{\text {ovf }}^{i} \\
\overline{A D E R}=\sum_{i=1}^{N} D E R^{i} / N
\end{gathered}
$$

Now $N_{\text {forg }}$ indicates quantity of forefront objects discovered by the moving object discovery technique, $N_{\text {ovf }}$ indicates the sum of really motion objects in starting video, and $N$ denotes the quantity of frames in video. We have actually chosen the Video 1, video 2 and Video 3 for the quantifiable examination since these are the most complicated videos and consist of several targets with differing dimension quantities. Table 9 provides the average detection error rate of each procedure for Video 1, video 2 and video 3.

Practical effects The exploration of movement object is a crucial advancement in computer visual images to develop different kind of structures, as an example, instinctive video surveillance and movement evaluation. These structures are made use of in a substantial range of usages, comprising resellers, residence computerisation, security, traffic observing, control applications, safety and more. In visual tracking security structures, the location of movement objects is a crucial task to recognize the useful little understanding from the video info, for instance, intrusion discovery, side-lined objects, and also traffic details collection and so forth. Object recognition gradually problem has a couple of necessary applications. This provides much better uncertainty that great using visional information and also offers to subsequently regard people also objects. In reseller's area, it split down the purchasing attributes of customers. In the traffic surveillance structure, it locates the flow of vehicles and distinguishes accidents. These are a part of making uses of objects recognition considerably trained.

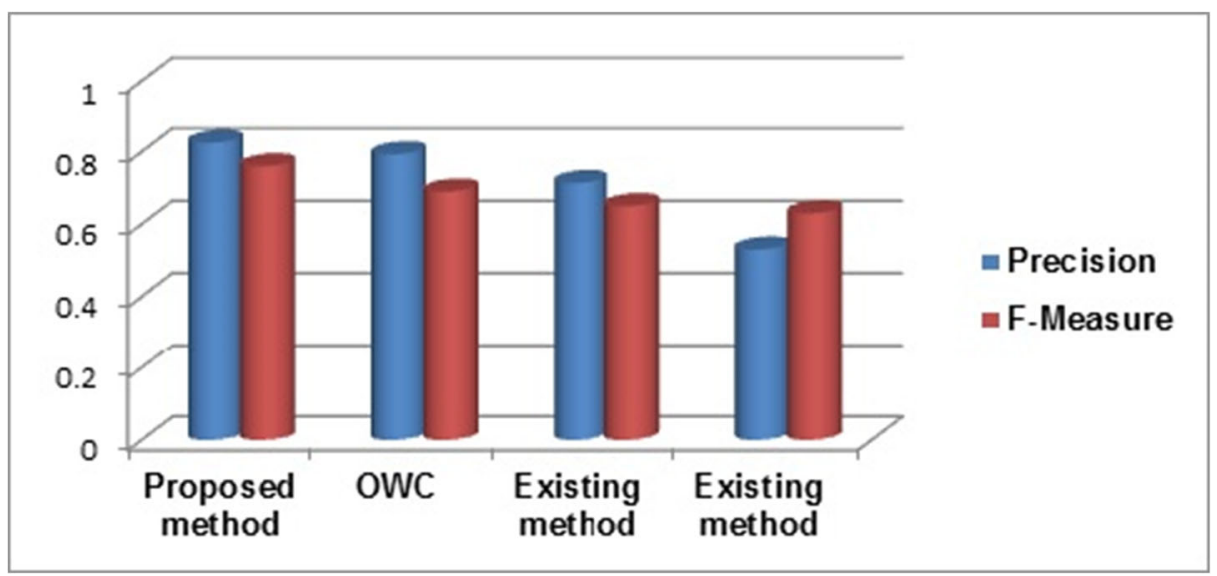

Fig. 7 Evaluation effects of the recommended method with previous methods 
Table 9 Contrast of detection error rates in percentage

\begin{tabular}{llll}
\hline Methods & \multicolumn{2}{l}{ Average detection error rate } & \\
\cline { 2 - 4 } & Video 1 & Video 2 & Video 3 \\
\hline Inter-frame differencing [29] & 26.09 & 30.03 & 32.16 \\
Combining method [29] & 10.53 & 18.27 & 17.53 \\
Proposed method & 6.42 & 11.24 & 12.32 \\
\hline
\end{tabular}

\section{Conclusion}

The suggested strategy effectively splits the background and forefront from the noise video collection using SKH- RKFCM procedure. The SKH procedure approximates the maximum centroid for executing the details clustering that is established the marginal fitness procedure attribute. Performance examination implemented taking advantage of both datasets validate that the recommended SKH outmatches all the previous strategy by conquering a very least value of MSE of 1.186 along with the large value of 0.9896 for f-measure, 0.9864 for rand coefficient, and 0.9092 for jaccord coefficient. Through the object discovery there are some problems alike embodied the local minima and initiation degree of level of sensitiveness. This produces RKFCM that does not implement well for the advancement of consistent clusters. The durable SKH methods integrate with refined kernel Fuzzy C means (RKFCM), to resolve the local minima and initiation of level of sensitiveness. Based upon SKH- RKFCM method the back-ground and also forefront objects are segregated. Moving objects are tracked by blob discovery which continuously traces the activity objects. Qualities are extracted from the improved frameworks in addition to organized in Tables 3 and 4. The mined features are obtained category which uses KNN, J48 and MLP classifiers in Table 5. The category end results of all 3 classifiers are identified and also prepared from Tables 6. The execution examination is end results are prepared in Table 7 which exposes the $\mathrm{J} 48$ classifier provides much better effectiveness in relation to improved discovery precision along with lessened FAR. The performance of the suggested method is analyzed via precision, recall, F-measure and accuracy. From the analytical end results, the suggested work surpasses than the state of artwork. Right here the recommended approach accomplishes optimal accuracy and also recall value for both PETS and also Hall monitor video when contrasted to the prevailing procedure. Here in the future, the experts may make use of a couple of various other objects tracking procedure to complete optimum effectiveness. In the prospect, we want to extend our work to track along with the discover object in a very stuffed location or invisibility of illumination variation and occlusion.

Open Access This article is licensed under a Creative Commons Attribution 4.0 International License, which permits use, sharing, adaptation, distribution and reproduction in any medium or format, as long as you give appropriate credit to the original author(s) and the source, provide a link to the Creative Commons licence, and indicate if changes were made. The images or other third party material in this article are included in the article's Creative Commons licence, unless indicated otherwise in a credit line to the material. If material is not included in the article's Creative Commons licence and your intended use is not permitted by statutory regulation or exceeds the permitted use, you will need to obtain permission directly from the copyright holder. To view a copy of this licence, visit http://creativecommons.org/licenses/by/4.0/. 


\section{References}

1. Ajala Funmilola A, Oke OA, Adedeji TO, Alade OM, Adewusi EA (2012) Fuzzy K-C-means clustering algorithm for medical image segmentation. J Informat Eng Appl 2(6)

2. Alihodzic A, Tuba M (2014) Improved bat algorithm applied to multilevel image thresholding. Sci World J 2014, Article ID 176718:16

3. Allin Christe S, Malathy K, Kandaswamy A (2010) Improved hybrid segmentation of brain MRI tissue and tumor using statistical features. ICTACT J Image Video Process 1(1). https://doi.org/10.21917 /ijivp.2010.0007

4. Arvanitidou MG, Tok M, Krutz AGA, Sikora T (2013) Motion based object segmentation using hysteresis and bidirectional inter-frame change detection in sequences with moving camera. Signal Process Image Commun 28:1420-1434

5. Borges PVK, Conci N, Cavallaro A (2013) Video based human behavior understanding: a survey. IEEE Trans Circ Syst Video Technol 23:1993-2008

6. Bouguessa M, Wang S, Sun H (2006) An objective approach to cluster validation. Pattern Recogn Lett 27(13):1419-1430

7. Choi W, Pantofaru C, Savarese S (2013) A general framework for tracking multiple people from a moving camera. IEEE Trans Pattern Anal Mach Intell 35:1577-1591

8. Cui D (2017) Application of whale optimization algorithm in reservoir optimal operation. Adv Sci Technol Water Resour 37(3):72-79

9. Datasets from <http://archive.ics.uci.edu/ml/>. Accessed 22 Mar 2019

10. Duan H, Zhao W, Wang G, Feng X (2012) Test-sheet composition using analytic hierarchy process and hybrid meta heuristic algorithm TS/BBO, Math Probl Eng. https://doi.org/10.1155/2012/712752

11. ElAziz M, Ewees AA, Hassanien AE (2017) Whale optimization algorithm and moth-flame optimization for multilevel thresholding image segmentation. Expert Syst Appl 83:242-256. https://doi.org/10.1016/j. eswa.2017.04.023

12. Faieghi MR, Delavari H, Baleanu D (2012) A novel adaptive controller for two-degree of freedom polar robot with unkown perturbations. Commun Nonlinear Sci 17(2):1021-1030. https://doi.org/10.1016/j. cnsns.2011.03.043

13. Farnad B, Jafarian A, Baleanu D (2018) A new hybrid algorithm for continuous optimization problem. Appl Math Model 55:652-673. https://doi.org/10.1016/j.apm.2017.10.001

14. Fei M, Li J, Liu H (2015) Visual tracking based on improved foreground detection and perceptual hashing. Neurocomputing 152:413-428

15. Fogel DB (2000) Evolutionary computation: toward a new philosophy of machine intelligence, second edn. IEEE Press, iscataway, NJ

16. Gandomi AH, Alavi AH (2011) Multi-stage genetic programming: A new strategy to nonlinear system modeling. Inf Sci 181:5227-5239

17. Gandomi AH, Alavi AH (2012) Krill herd: a new bio-inspired optimization algorithm. Commun Nonlinear Sci Numer Simul 17:4831-4845

18. Gandomi AH, Yang X-S, Talatahari S, Alavi AH (2013) Meta heuristic applications in structures and infrastructures. Elsevier, Waltham

19. Gao H, Xu W, Sun J, Tang Y (2010) Multilevel thresholding for image segmentation through an improved quantum-behaved particle swarm algorithm. IEEE Trans Instrum Meas 59:934-946

20. Goldberg DE (1998) Genetic algorithms in search, Optimization and Machine Learning. Addison-Wesley, NewYork

21. He H, Tan Y (2012) A two-stage genetic algorithm for automatic clustering. Neurocomputing 81:49-59

22. Hong X, Chen S (2012) The system identification and control of Hammerstein system using nonuniformrational B-spline neural network and particle swarm optimization. Neurocomputing 82:216-223

23. Horng MH (2010) Multilevel minimum cross entropy threshold selection based on the honey bee mating optimization. Expert Syst Appl 37(6):4580-4592

24. Horng $\mathrm{MH}$ (2011) Multilevel thresholding selection based on the artificial bee colony algorithm for image segmentation. Expert Syst Appl 38(11):13785-13791

25. Horng M-H, Liou R-J (2011) Multilevel minimum cross entropy threshold selection based on the firefly algorithm. Expert Syst Appl 38(12):14805-14811

26. Horng M-H, Jiang T-W, Chen J-Y (2009) Multilevel Minimum Cross Entropy threshold Selection based on Honey Bee Mating Optimization. In: Proceedings of the International Multi Conference of Engineers and Computer Scientists, Hong Kong 1:978-988. https://doi.org/10.1016/j.eswa.2009.12.050

27. Hou L, Wan W, Lee K-H, Hwang J-N, Okopal G, Pitton J (2015) Deformable multiple-kernel based human tracking using a moving camera. In: Acoustics, Speech and Signal Processing (ICASSP), 2015 IEEE International Conference pp. 2249-2253. https://doi.org/10.1109/ICASSP.2015.7178371 
28. Hsieh T-J, Hsiao H-F, Yeha W-C (2012) Mining financial distress trend data using penalty guided support vector machines based on hybrid of particle swarm optimization and artificial bee colony algorithm. Neurocomputing 82:196-206

29. Hu W-C, Chen C-H, Chen T-Y, Huang D-Y, Wu Z-C (2015) Moving object detection and tracking from video captured by moving camera. J Vis Commun Image Represent 30:64-180

30. Jadhav AN, Gomathi N (2016) Kernel-based exponential grey wolf optimizer for rapid centroid estimation in data clustering. J Technol 78(11):65-74

31. Javed S, Jung SK, Bouwmans T (2017) Improving OR-PCA via smoothed spatially-consistent low-rank modeling for background subtraction. In: Proceedings of the 32nd ACM Symposium on Applied Computing (SAC-17) pp. 89-94. https://doi.org/10.1145/3019612.3019637

32. Jiang Y, Tsai P, Yeh W-C, Cao L (2017) A honey-bee mating based algorithm for multilevel image segmentation using Bayesian theorem. Appl Soft Comput 52:1181-1190

33. Khatib W, Fleming P (1998) The stud GA: a mini revolution? In: Eiben A, Back T, Schoenauer M, Schwefel H (eds) Proceedings of the 5th International Conference on Parallel Problem Solving from Nature. Springer-Verlag, New York, USA, pp 683-691

34. Kim SW, Yi KYKM, Kim SJ, Choi JY (2013) Detection of moving objects with a moving camera using non-panoramic background model. Mach Vis Appl 24:1015-1028

35. Lai CC, Tseng DC (2004) A hybrid approach using Gaussian smoothing and genetic algorithm for multilevel thresholding. Int J Hybrid Intell Syst 1(3):143-152

36. Le Capitaine H, Frelicot C (2011) A fast fuzzy c-means algorithm for color image segmentation. In: Proceedings of the 7th conference of the European Society for Fuzzy Logic and Technology (EUSFLAT11) pp. 1074-1081. https://doi.org/10.2991/eusflat.2011.9

37. Li Y, Li G (2009) Fuzzy C-means cluster segmentation algorithm based on modified membership. In: Proceedings 6th International Symposium on Neural Networks, ISNN 2009 Wuhan, China pp. 135-144

38. Li X, Li L, Lu H, Chen D, Liang Z (2003) Inhomogeneity correction for magnetic resonance images with fuzzy C-mean algorithm. Proc SPIE Int Soc Opt Eng 5032:995-1005

39. Lu H-C, Tsai C-H, Chang M-H (2012) Parameter estimation of fuzzy neural network controller based on a modified differential evolution. Neurocomputing 89:178-192

40. Mahalingam T, Subramoniam (2019) A hybrid gray wolf and genetic whale optimization algorithm for efficient moving object analysis. Accepted in Multimedia Tools and Applications 78:26633-26659. https://doi.org/10.1007/s11042-019-07768-x

41. Maulik U, Mukhopadhyay A (2010) Simulated annealing based automatic fuzzy clustering combined with ANN classification for analyzing microarray data. Comput Oper Res 37:1369-1380. https://doi.org/10.1016 j.cor.2009.02.025

42. Minematsu T, Uchiyama H, Shimada A, Nagahara H, Taniguchi R-I (2015) Evaluation of foreground detection methodology for a moving camera. Frontiers of Computer Vision (FCV), 2015 21st Korea-Japan Joint 700 Workshops on, IEEE. pp. 1-4. https://doi.org/10.1109/FCV.2015.7103752

43. Mirjalili S, Lewis A (2016) The whale optimization algorithm. Adv Eng Softw 95:51-67

44. Mlakar U, Potoc`nik B, Brest J (2016) A hybrid differential evolution for optimal multilevel image thresholding. Expert Syst Appl 65:221-232

45. Muangkote N, Sunat K, Chiewchanwattana S (2017) Rr-cr- IJADE: an efficient differential evolution algorithm for multilevel image thresholding. Expert Syst Appl 90:272-289

46. Sathya PD, Kayalvizhi R (2011) Optimal multilevel thresholding using bacterial foraging algorithm. Expert Syst Appl 38:15549-15566

47. Sezgin M, Sankur B (2004) Survey over image thresholding techniques and quantitative performance evaluation. J Electron Imaging 13:146-165

48. Sharma S, Buddhiraju KM (2018) Spatial-spectral ant colony optimization for hyperspectral image classification. Int J Remote Sens 39(9):2702-2717. https://doi.org/10.1080/01431161.2018.1430403

49. Silva V, Khati W, Fleming P (2005) Performance optimization of gas turbine engine. Eng Appl Artif Intell 18:575-583

50. Simpson AJR (2015) On-the-fly learning in a perpetual learning machine. arXiv preprint arXiv:1509.00913

51. Song J-H, Cong W, Li J (2017) A fuzzy C-means clustering algorithm for image segmentation using nonlinear weighted local information. J Inf Hiding Multimedia Signal Process 8(3):578-588

52. Talatahari S, Kheirollahi M, Farahmandpour A, Gandomi H (2013) A multi-stage particle swarm for optimum design of truss structures. Neural Comput \& Applic 23:1297-1309. https://doi.org/10.1007 /s00521-012-1072-5

53. Thangaraj M, Monikavasagom S (2019) A competent frame work for efficient object detection, tracking and classification. Wirel Pers Commun 107(2):939-957. https://doi.org/10.1007/s11277-019-06310-4 
54. Tsai C-Y, Chiu C-C (2007) An efficient feature selection approach for clustering: using a Gaussian mixture model of data dissimilarity. In: Proceedings of international conference on computational science and its applications. Springer, Berlin Heidelberg, pp 1107-1118

55. Wang G, Guo L, Gandomi AH, Cao L, Alavi AH, Duan H, Li J (2013) Lévy-flight krill herd algorithm. Math Probl Eng 1-14. https://doi.org/10.1155/2013/682073

56. Wei Y, McNicholas PD (2015) Mixture model averaging for clustering. Adv Data Anal Classif 9(2):197217

57. Wu Y, Lim J, Yang M-H (2015) Object tracking benchmark. IEEE Trans Pattern Anal Mach Intell 37(9): 1834-1848

58. Yang X-S, Talatahari S, Gandomi AH, Alavi AH (2013) Meta heuristics in water, geotechnical and transport engineering. Elsevier, Waltham

59. Ye Z, Zheng Z, Yu X, Ning X (2006) Automatic threshold selection based on ant colony optimization algorithm. In: proceedings of IEEE International conference on neural networks and brain, Beijing, pp. 728732. https://doi.org/10.1109/ICNNB.2005.1614730

60. Yin PY, Chen LH (1997) A fast iterative scheme for multilevel thresholding methods. Signal Process 60(3): 305-313

61. Zhang D-Q, Chen S-C, Pan Z-S, Tan K-R (2003) Kernel-based fuzzy clustering incorporating spatial constraints for image segmentation. Proc Int Conf Mach Learn Cybern 4:2189-2192

62. Zhang C, Ouyang D, Ning J (2010) An artificial bee colony approach for clustering. Exp Syst Applic 37: 4761-4767. https://doi.org/10.1016/j.eswa.2009.11.003

Publisher's note Springer Nature remains neutral with regard to jurisdictional claims in published maps and institutional affiliations.

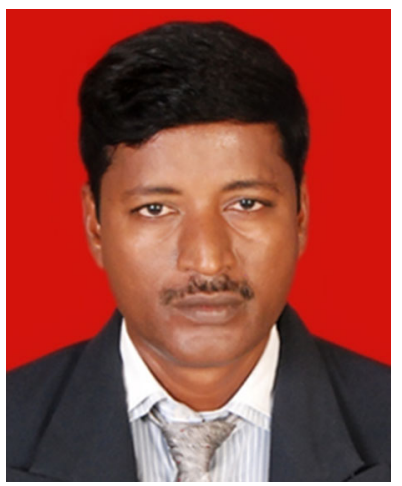

T. Mahalingam Designation:Ph.D., Scholar,Ssathyabama university, Chennai, Tamil Nadu, India. 